\title{
Writing and reading of single magnetic domain per bit perpendicular patterned media
}

\author{
Mladen Todorovic and Sheldon Schultz \\ Department of Physics and Center for Magnetic Recording Research, University of California, San Diego, \\ La Jolla, California 92093-0319
}

\author{
Joyce Wong and Axel Scherer \\ Department of Electrical Engineering, California Institute of Technology, Pasadena, California 91125
}

(Received 15 January 1999; accepted for publication 26 February 1999)

\begin{abstract}
By fabricating patterned media with a large number of nanoscale single domain magnetic particles embedded in a nonmagnetic substrate, and by writing the magnetization for each of these particles in a desired direction, nonvolatile magnetic storage of information could reach densities much higher than what is currently thought possible for longitudinal continuous media. We have fabricated high aspect ratio perpendicular nickel columnar nanoparticles embedded in a hard $\mathrm{Al}_{2} \mathrm{O}_{3} / \mathrm{GaAs}$ substrate. We show that the magnetization states of the individual magnets can be controlled by demonstrating that prototype patterned "single magnetic domain per bit" data tracks can be written and read back using current magnetic information storage technology. (C) 1999 American Institute of Physics. [S0003-6951(99)00717-2]
\end{abstract}

Current commercial magnetic disk data storage density employing a continuous film and a longitudinal bit format is at $\sim 5$ Gbits/in. ${ }^{2}$. Extensive recent theoretical analysis ${ }^{1,2}$ supports $\sim 40$ Gbits/in. ${ }^{2}$, but casts severe doubts on achieving stable storage at densities of $100 \mathrm{Gbits} / \mathrm{in} .^{2}$ due to the thermal instability of the distribution in size of the individual magnetic grains which behave as superparamagnetic particles. Two high density magnetic storage schemes, both based on lithographically defined single domain magnet arrays, have been proposed to solve the superparamagnetic limit problem. For practical media these designs must maintain the necessary signal to noise ratio, while avoiding the onset of the superparamagnetic limit for areal densities beyond 100 Gbits/in. ${ }^{2}$.

In the longitudinal patterned single domain per bit storage format, ${ }^{3}$ the magnetic medium is comprised of lithographically defined longitudinal single domain thin film magnetic elements. The fabrication, magnetic characterization, ${ }^{4,5}$ and manipulation of these types of magnets with local magnetic probes $^{6-8}$ have been demonstrated, and research is currently being conducted to better understand their physical properties and improve the crystalline anisotropy. The second proposed design for patterned single magnetic domain per bit storage is the perpendicular version, where the particle long axis is oriented perpendicular to the medium surface. 9 In this "patterned media" format, the magnetization direction along the easy axis of each individual single domain particle represents the " 1 " ' or " 0 " of the stored data bit. The fabrication and characterization of arrays of individual magnetic single domain columnar particles with a size and spacing that will support bit densities of $\sim 100$ Gbits/in. ${ }^{2}$ have been demonstrated. ${ }^{9-11}$ The effects of local magnetic probes on these particles have been observed, ${ }^{12}$ but effective nonvolatile magnetic storage including controlled writing has not yet been demonstrated.

Recent effort has gone into the fabrication and characterization of structures compatible with current commercial technology. ${ }^{13,14}$ Sensing of the magnetic fields from single domain particles by a commercial magnetoresistive sensor has been previously demonstrated, ${ }^{15}$ however, it was discovered that the fields from the current in the read sensor were switching the particle magnetization during the reading process, thereby eliminating any possibility of reliable nondestructive data storage. ${ }^{15}$ In this letter, we demonstrate the successful writing and reading of individual high aspect ratio single domain particles, using a commercial inductive and magnetoresistive head, respectively, thereby demonstrating model patterned media data tracks. This demonstration bridges the gap between the fabrication of patterned media, and its potential application in today's magnetic recording industry by using current read/write technology.

After reexamining previous fabrication techniques, we have focused on improving the coercivity of single domain particles by increasing their aspect ratio and therefore their shape anisotropy. ${ }^{16}$ The procedure for the fabrication of high aspect ratio nickel columns is schematically shown in Fig. 1. We use vector scanned electron beam lithography to define our structures on the poly(methylmethacrylate) (PMMA) coated AlAs/GaAs substrate. After development in a 3:7 cellulose-methanol mixture, the square array of dots is then transferred into the AlAs/GaAs substrate using $\mathrm{Cl}_{2}$ assisted ion beam etching. Once the holes in the GaAs substrate are defined, wet thermal oxidation follows immediately to convert $\mathrm{AlAs}$ into $\mathrm{Al}_{2} \mathrm{O}_{3}$. This robust $\mathrm{Al}_{2} \mathrm{O}_{3}$ layer then acts as an etch mask for further chemically assisted ion beam etching (CAIBE) to amplify the dot array pattern into the GaAs substrate in the perpendicular direction. This process takes advantage of the highly selective etching and masking properties of $\mathrm{GaAs}$ and $\mathrm{Al}_{2} \mathrm{O}_{3}$, respectively, and allows us to create high aspect ratio holes in the substrate. The etched holes are then filled with nickel using electroplating, resulting in single domain magnetic particles embedded in the GaAs substrate. Finally, overplated nickel mushrooms are removed by polishing, which creates a smooth surface for further magnetic analysis. A scanning electron microscope image of the square array of empty holes in $\mathrm{Al}_{2} \mathrm{O}_{3}$ before plating, and the same array filled with nickel single domain particles, is shown in Figs. 2(a) and 2(b), respectively. The columns are 
(a) MBE growth and mask deposition

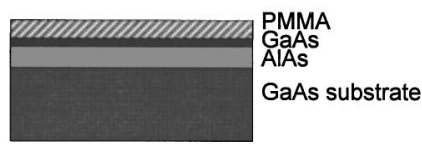

(b) Electron beam lithography

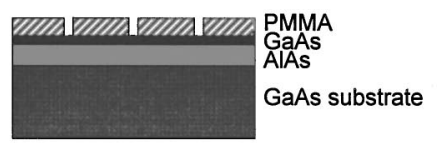

(c) Chemically assisted ion beam etch

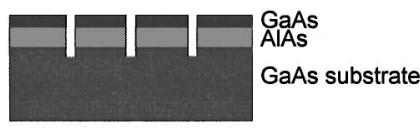

(d) Wet thermal oxidation

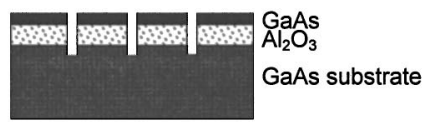

(e) Chemically assisted ion beam etch

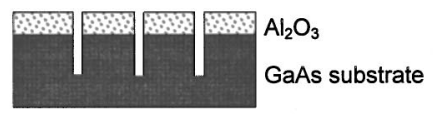

(f) Electroplating and Polishing

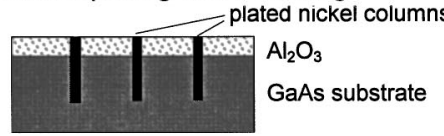

FIG. 1. Schematic diagram of the fabrication procedure of high aspect ratio nickel columns embedded in an $\mathrm{Al}_{2} \mathrm{O}_{3} / \mathrm{GaAs}$ substrate.

$170 \mathrm{~nm}$ in diameter, approximately $900 \mathrm{~nm}$ high, and $2 \mu \mathrm{m}$ apart. In Fig. 2(c), we show a $12 \mu \mathrm{m} \times 12 \mu \mathrm{m}$ scan of the same sample, imaged using magnetic force microscopy. ${ }^{17}$

We use the technique of scanning magnetoresistance microscopy $(\mathrm{SMRM})^{18}$ to evaluate whether perpendicular patterned media can be read and written with current technology. In this technique, a commercial magnetoresistive head is raster scanned in contact over the sample mounted on a high-resolution linearized piezoelectric scanning stage. The magnetoresistive sensor used in this work has a width of 1.9 $\mu \mathrm{m}$, with shield spacing of $240 \mathrm{~nm}$, while the poles of the write element are $200 \mathrm{~nm}$ apart and $2.2 \mu \mathrm{m}$ wide. The SMRM as well as the current magnetic disk storage technology, requires that the sample surface be robust and smooth, both of which are satisfied by our sample fabrication. We orient the sample so that the square array of columns is at $45^{\circ}$ to the SMRM scan direction, in order to maximize the separation between the particles that we attempt to read and write. Magnetoresistive bias current of $7 \mathrm{~mA}$ was used in all the SMRM images presented in this letter. In all the images, the columns appear as stripes due to the finite width of the magnetoresistive read sensor.

Figure 3 is a collection of images demonstrating that the model single column per bit patterned media data tracks can be controllably written, and then read using commercial inductive write and magnetoresistive read elements, respectively. For writing, we use the perpendicular components of the fringing field from the write element poles to orient the columns in the desired direction. A current of $5 \mathrm{~mA}$ through Downloaded 18 Dec 2005 to 131.215.240.9. Redistribution subject
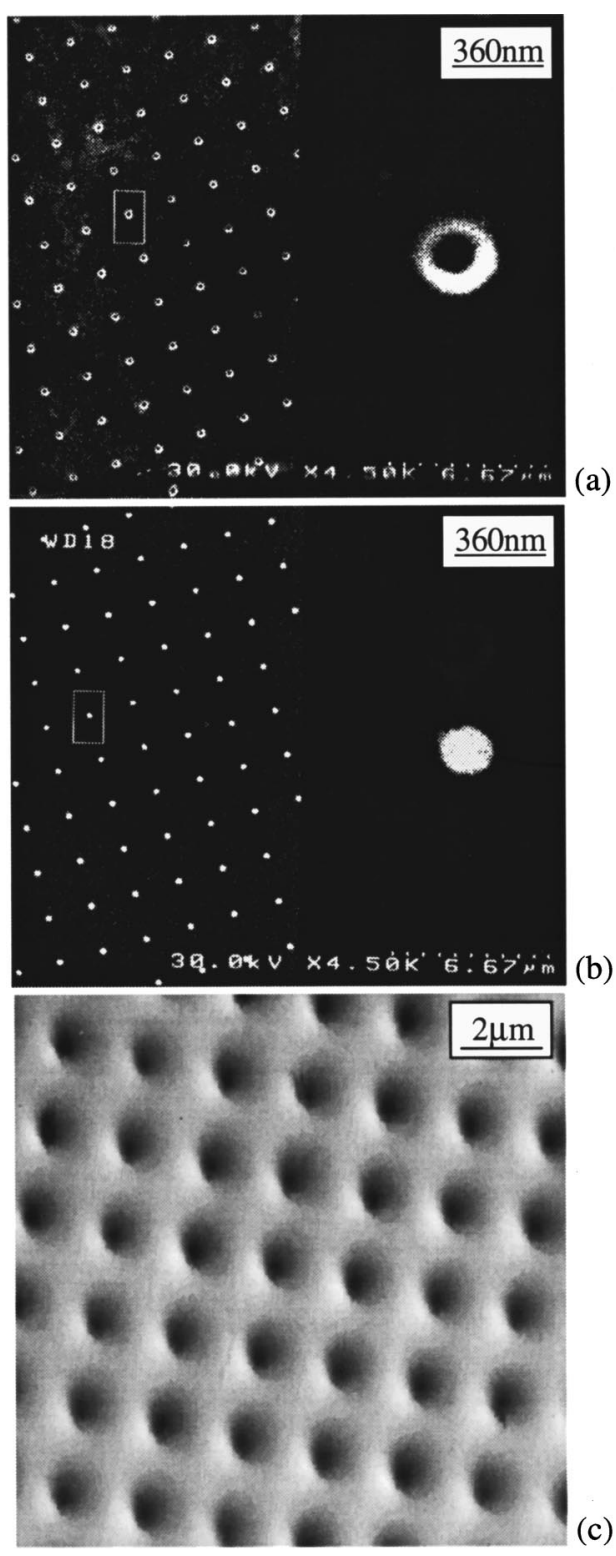

(c)

FIG. 2. SEM images of (a) an array of etched holes in $\mathrm{Al}_{2} \mathrm{O}_{3} / \mathrm{GaAs}$ before electroplating nickel (left) and a $10 \times$ enlargement of one of the etched holes (right), (b) the same array filled with nickel by electroplating (left) and a $10 \times$ enlargement of one of the plated nickel columns (right). A $12 \mu \mathrm{m} \times 12 \mu \mathrm{m}$ magnetic force microscopy image of the array is shown in part (c).

the write element is sufficient to switch the magnetization of the columns. Six different configurations of three adjacent tracks are shown. In Fig. 3(a), the columns in all three tracks are oriented in the up direction and appearing white, while in Fig. 3(b), all are in the down direction and appearing black. These two images demonstrate that the columns can have two magnetic states and can be read nondestructively. In Fig. 3(c), the columns in the center track are all in the up state, while the columns in the side tracks are in the down state. The next three images show patterns with more realistic appearance of patterned media data tracks. In Fig. 3(d), the center track has every third magnetic domain written up, while the side tracks have all the columns written down. Figure 3(e) shows the center track with columns written in an alternating up/down sequence, while the side tracks are all down. In Fig. 3(f), all three tracks are written in the alternato AIP license or copyright, see http://apl.aip.org/apl/copyright.jsp 
(a)
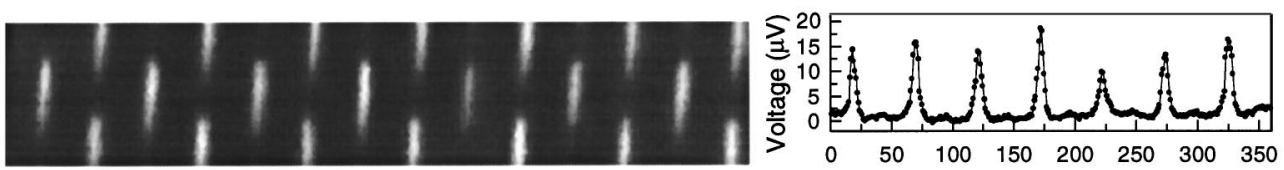

(b)
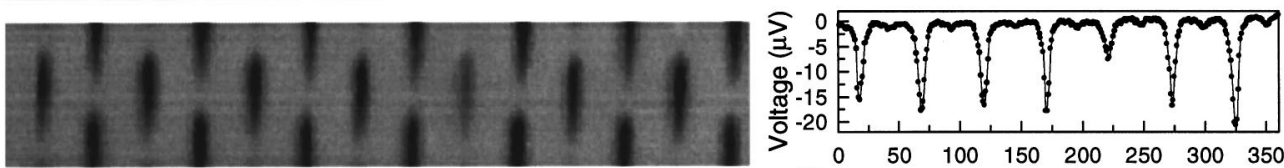

c)
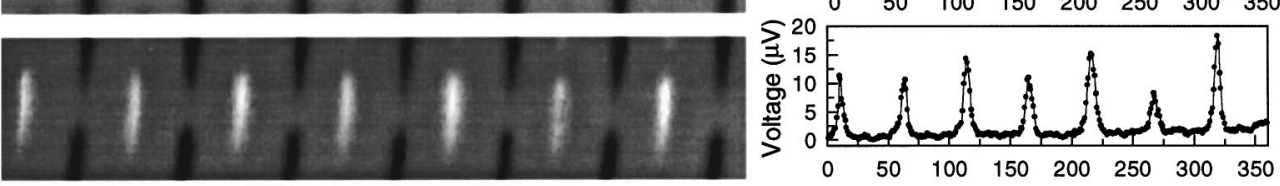

(d)
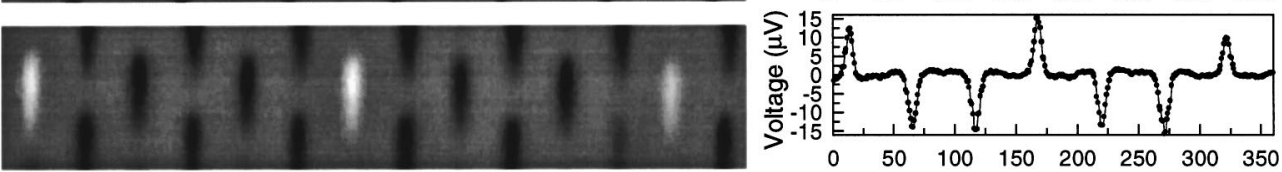

(e)
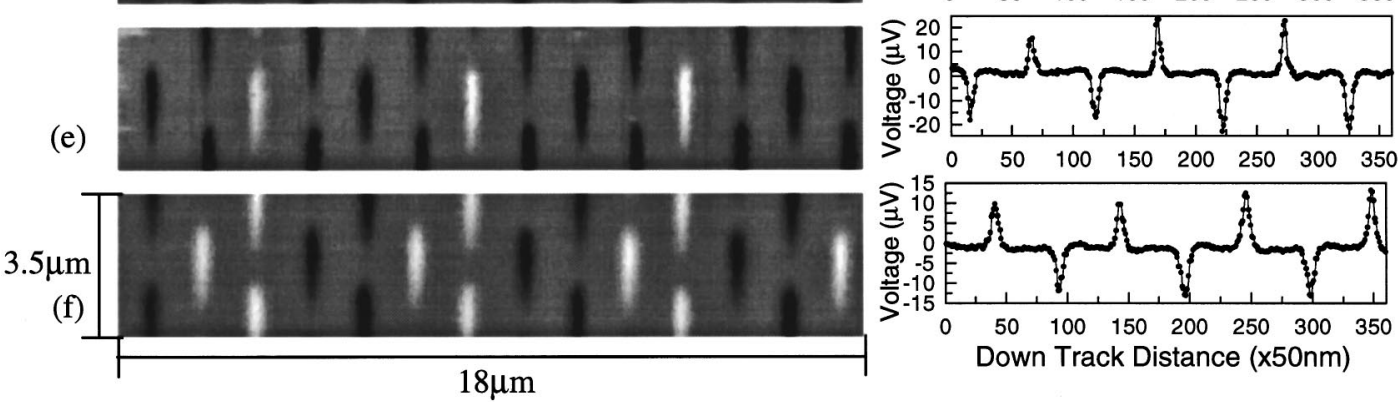

FIG. 3. A collection of SMRM images, demonstrating controllable writing of single column per bit perpendicular patterned media data tracks, is shown on the left side. The line scans of the center data tracks are shown on the right side.

ing up/down sequence. The single line scans, next to each gray scale image, show the magnetoresistive voltage signal from the center data track configuration. These voltages represent the read-back signal that would be used for further signal processing and data retrieval in a patterned media storage system.

The signal amplitude is an order of magnitude smaller than typically observed in continuous thin film magnetic recording systems. This is expected, since the effective magnetic charge of a single magnetic domain that the sensor detects is approximately an order of magnitude smaller than the effective magnetic charge that the larger (i.e., $2 \mu \mathrm{m}$ ) data tracks provide. ${ }^{15}$ Simulations of the magnetoresistive sensor spatial response using the Potter approximation ${ }^{19}$ agree well with the linewidth of the signal that we observe. The signal amplitude is dependent on the distance between the magnetoresistive sensor and the column surface, and the variation that we observe corresponds to a $20 \mathrm{~nm}$ height difference, which is to be expected in our SMRM experimental arrangement. We believe that we are observing the near maximum value of the signal that is theoretically expected from a uniformly magnetized single domain particle, based on the effective magnetic charge comparison. Since the magnetic field sensors used in the magnetic storage industry are continually advancing towards higher sensitivities and smaller track widths, the work that we present here will be accordingly scaled to the smaller diameter columns in the future.

The authors would like to thank George Kassabian, Sam Hobbs, and Mark Vojkovich for technical help, J. Smyth and M. Re for providing them with the MR heads, Chuan-Cheng Downloaded 18 Dec 2005 to 131.215.240.9. Redistribution subject
Cheng for helpful discussions, and Reginald Lee for the growth of the MBE samples. This work was supported by the Center for Magnetic Recording Research, ARO, and NSF (MRSEC) Grant No. DMR-94-00439.

${ }^{1}$ S. H. Charap, P. L. Lu, and Y. He, IEEE Trans. Magn. 33, 1 (1997).

${ }^{2}$ H. N. Bertram, H. Zhou, and R. Gustafson, IEEE Trans. Magn. 34, 4 (1998).

${ }^{3}$ R. L. White, R. M. N. New, and R. F. W. Pease, IEEE Trans. Magn. 33, 1 (1997)

${ }^{4}$ J. F. Smyth, S. Schultz, D. R. Fredkin, D. P. Kern, S. A. Rishton, H. Schmidt, M. Cali, and T. R. Koehler, J. Appl. Phys. 69, 8 (1991).

${ }^{5}$ R. M. H. New, R. F. W. Pease, and R. L. White, J. Magn. Magn. Mater. 155, 140 (1996).

${ }^{6}$ G. A. Gibson, J. F. Smyth, S. Schultz, and D. P. Kern, IEEE Trans. Magn. 27, 5187 (1991).

${ }^{7}$ L. Kong, L. Zhuang, and S. Y. Chou, IEEE Trans. Magn. 33, 5 (1997).

${ }^{8}$ M. Kleiber, F. Kummerlen, M. Lohndorf, A. Wadas, D. Weiss, and R. Wiesendanger, Phys. Rev. B 58, 9 (1998).

${ }^{9}$ S. Y. Chou, M. S. Wei, P. R. Krauss, and P. B. Fisher, J. Appl. Phys. 76, 10 (1994)

${ }^{10}$ A. D. Kent, S. von Molnar, S. Gider, and D. D. Awschalom, J. Appl. Phys. 76, 10 (1994).

${ }^{11}$ W. Xu, J. Wong, C. C. Cheng, R. Johnson, and A. Scherer, J. Vac. Sci. Technol. B 13, 6 (1995).

${ }^{12}$ G. Meier, M. Kleiber, D. Grundler, D. Heitmann, and R. Wiesendanger, Appl. Phys. Lett. 72, 17 (1998).

${ }^{13}$ S. Y. Chou, P. R. Krauss, and L. Kong, J. Appl. Phys. 79, 8 (1996).

${ }^{14}$ R. O'Barr, S. Y. Yamamoto, S. Schultz, W. Xu, and A. Scherer, J. Appl. Phys. 81, 8 (1997).

${ }^{15}$ S. Y. Yamamoto, R. O'Barr, S. Schultz, and A. Scherer, IEEE Trans. Magn. 33, 5 (1997).

${ }^{16}$ J. Wong, A. Scherer, M. Todorovic, and S. Schultz, J. Appl. Phys. 85, 8 (1999).

${ }^{17}$ Y. Martin and H. K. Wickramasinghe, Appl. Phys. Lett. 50, 1455 (1987).

${ }^{18}$ S. Y. Yamamoto and S. Schultz, Appl. Phys. Lett. 69, 21 (1996).

${ }^{19}$ R. Potter, IEEE Trans. Magn. 10, 502 (1974).

o AIP license or copyright, see http://apl.aip.org/apl/copyright.jsp 